Article

\title{
Mixing Time-Experimental Determination and Applications to the Modelling of Crystallisation Phenomena
}

\author{
Dragan D. Nikolić *, Giuseppe Cogoni and Patrick J. Frawley \\ Synthesis and Solid State Pharmaceutical Centre, University of Limerick, Limerick, Ireland; \\ giusecogoni@gmail.com (G.C.); Patrick.Frawley@ul.ie (P.J.F.) \\ * Correspondance: dnikolic@daetools.com
}

\begin{abstract}
Performing optimisation and scale-up studies of crystallisation systems requires accurate and computationally efficient mathematical models. The assumption of the ideal mixing conditions in batch reactors typically produce inaccurate results while the computational expense of CFD models is still prohibitively high. Therefore, in this work, a new intermediary approach is proposed that takes into account the non-ideal mixing conditions in the reactor and requires less computational resources than full CFD simulations. Starting with the Danckwerts concept of the intensity of segregation, an analogy between its application to chemical reactions and the kinetics of the crystallisation phenomena (such as nucleation and growth) has been made. As a result, the modified kinetics expressions have been derived which incorporate the effect of non-idealities present in stirred reactors. This way, based on the experimental measurements of the mixing time using the Laser Induced Fluorescence (LIF) technique, computationally more efficient mathematical models can be developed in two ways: (1) the accurate semi-empirical correlations are available for standard mixing configurations with the most often used types of impellers, (2) CFD simulations can be utilised for estimation of the mixing time; in this case it is necessary to simulate only the mixing process. The benefits offered by the LIF experimental technique have been demonstrated and some frequent problems in its application analysed. The mixing time results for configurations with and without baffles for three types of impellers and four different rotational speeds have been presented. The false shorter mixing times in the nonbaffled configurations have been observed and this phenomena explained by the existence of two segregated zones in the reactor and confirmed by additional experiments. The precise measurements in these cases have been shown as difficult using the LIF technique, particularly for higher rpms. The experimental data has been compared to the preliminary simulation results obtained from the Smoothed Particle Hydrodynamics method and the standard k- $\varepsilon$ turbulence model with the modest success. The shortcomings of the SPH model have been recognized and the directions for the future work discussed.
\end{abstract}


Keywords: mixing time; LIF; CFD; SPH; stirred tank

\section{Introduction}

Mixing is a very important aspect of a crystalliser design, since it is responsible for an efficient mass, heat transport and transport of particles throughout the crystalliser. The local hydrodynamics conditions affect to a great extent almost all phenomena in the crystalliser (such as primary and secondary nucleation, growth, aggregation, agglomeration and breakage). For these reasons, computational fluid dynamics (CFD) simulations are often employed to capture the most important information about the fluid flow. However, one of the major problems is the computational expense of CFD models due to a large number of equations that need to be solved and due to a large difference in time resolution of up to ten orders of magnitude in some cases: a time frame of a typical crystallisation process is several hours compared to a time step necessary to achieve reasonable accuracy in the range of microseconds. While some methods for rapid prediction of the fluid flow in stirred reactors have been developed in our group (Nikolic and Frawley, 2015) this approach is still computationally inefficient for scale-up/optimisation studies: a typical problem still requires two to three days simulation time per hour of crystallisation process time, with the currently available GPGPU (general purpose graphics processing units) hardware. Although the latest generation of GPU cards brings almost a double increase in performance - the overall picture has not been changed significantly.

\subsection{Definitions of mixing quality}

Although a number of definitions of the mixing quality have been proposed in the literature, no single definition accurately and clearly describes the full range of problems in the field of industrial mixing. Some of the proposed approaches are reviewed.

\subsubsection{Intensity of segregation}

In chemical reactor engineering, the assumption is usually made that only the mean concentration needs to be considered; in reality, concentration values fluctuate about a mean, and in some cases these fluctuations must be considered in detail (Ingham et al., 2007). The concept of the segregation and its meaning to chemical reactors was first described by Danckwerts $(1952 ; 1958)$. The intensity of segregation is a measure of the difference in 
concentration between the purest concentration of component $\mathrm{A}$ and the purest concentration of component B in the surrounding fluid (Paul et al., 2004). The intensity of segregation is a parameter that varies between one and zero, and is presented schematically in Fig. 1.

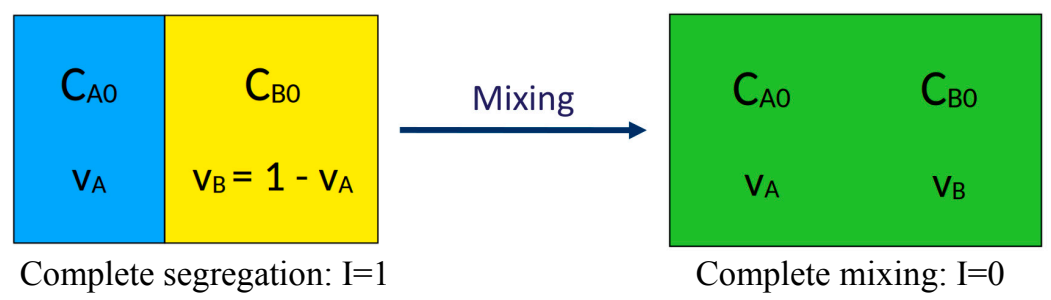

Figure 1. The concept of segregation (Ingham et al., 2007)

The intensity of segregation can be described in terms of concentration fluctuations illustrated in Fig. 2.

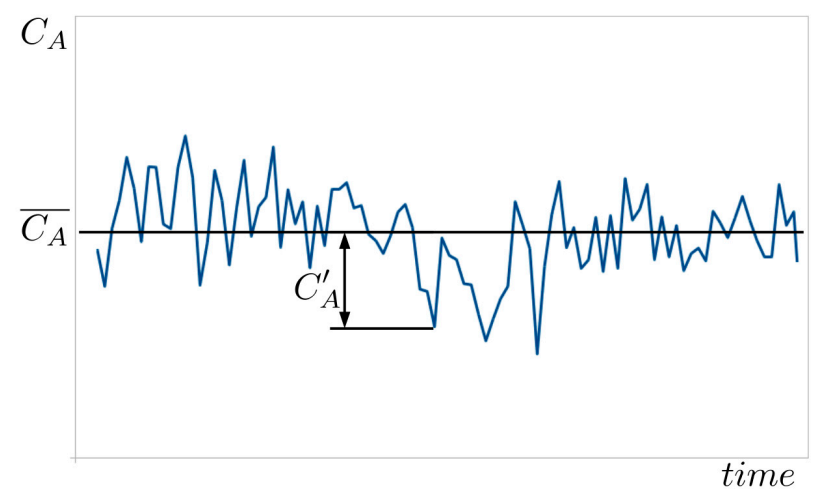

Figure 2. Time-variant turbulent fluctuations of concentration $\left(C^{\prime} A\right)$ about a mean value (Ingham et al., 2007)

The unmixedness can be characterized by the mean of the square of the fluctuations or concentration variance:

$$
\overline{C_{A}^{\prime 2}}=\overline{\left(\overline{C_{A}}-C_{A}^{\prime}\right)^{2}}
$$

To quantify the "state of unmixedness" Danckwerts introduced the concept of the intensity of segregation $(I)$, which is calculated in terms of the mean square of the fluctuations, as (Ingham et al., 2007):

$$
I(t)=\frac{\overline{C_{A}^{\prime 2}}}{\overline{C_{A 0}^{\prime 2}}}
$$


where the subscript 0 denotes the initial or feed value. In a segregated system or in a system without a complete mixing, the rate of any reaction between the reactants could obviously be influenced by the rate of mixing, as measured by the change in $I$. Starting with the general macroscopic mass balance equation for segregation or unmixedness in a non-reactive batch reactor system where only the accumulation and dissipation terms are important (Ingham et al., 2007), we get:

$$
V \frac{d\left(\overline{C_{A}^{\prime 2}}\right)}{d t}=-\frac{1}{\tau_{m}} \overline{C_{A}^{\prime 2}} V
$$

With the initial value $\overline{C_{A 0}^{\prime}}$ integration gives:

$$
\frac{\overline{C_{A}^{\prime 2}}}{\overline{{C_{A 0}^{\prime}}^{2}}}=I(t)=e^{-\frac{t}{\tau_{m}}}
$$

where $t$ is time elapsed in the process and $\tau_{m}$ is mixing time. This equation predicts that the intensity of segregation decays with time in the batch reactor. A similar equation can be derived for a steady-state plug flow system where the mean residence time should be used instead of $t$ (Ingham et al., 2007). However, all non-idealities are lumped into a single parameter $\tau_{m}$ and therefore in this approach it is not possible to identify what is the source of the non-idealities.

\subsubsection{Multi-dimensional definition of segregation}

According to Kukukova et al. (2009) several mixing objectives occur simultaneously in a single application and can be grouped into three categories: (i) blending of miscible liquids, (b) multiphase mixing with at least one of several objectives: "just contacted", completely distributed throughout the vessel, size reduction, or mass transfer, and (c) reaction (homogeneous or heterogeneous). The analysis of these applications, reveals three variables directly related to mixing: (a) a reduction in the segregation of concentration, (b) a reduction in the scale of segregation, and (c) a mixing time scale. Consequently, Kukukova et al. (2009) defined segregation as being composed of three separate dimensions: (i) the intensity of segregation quantified by the normalized concentration variance (concentration scale), (ii) the scale of segregation or clustering (length scale), and (iii) the exposure or the potential to reduce segregation (rate of change of segregation). The first dimension focuses on the instantaneous concentration variance; the second on the instantaneous length scales in the 
mixing field; and the third on the driving force for change, i.e. the mixing time scale, or the instantaneous rate of reduction in segregation.

\subsection{Definitions of the mixing time - uniformity criteria}

In this work the concept of the intensity of segregation as a function of the mixing time (equation 4) has been adopted. Although the mixing time is widely used in process industries, there is no standard definition for it in the literature. Besides the reactor design and the operating conditions in it, the measured blend time also depends on the measurement locations, the size and the number of the probes, and the final condition of mixing. One of the earliest attempts is by Kramers et al. (1953) who measured the time history of injected tracer solution into the batch stirred tank reactor. They defined mixing time as the interval from the time the tracer is injected to the time when the tracer concentration at the specified location is within $0.1 \%$ of the final mean concentration in the tank. They found that the total impeller revolutions $\left(N \tau_{m}\right)$ is approximately constant and developed the correlations for $N \tau_{m}$ as a function of the ratio of impeller diameter to tank diameter and the degree of homogeneity. Ranade et al. (1991) reviewed five different definitions and showed that each would give a different value for the same mixing process. Different blend times have been used in literature for different degrees of homogeneities, such as $\tau_{m 90}$ for $90 \%, \tau_{m 95}$ for $95 \%$, and $\tau_{m 99}$ for $99 \%$ homogeneity. The percentage is the ratio of the concentration at the measuring point to the final mean concentration. It was also reported in literature that the measured blend time varies with the spatial locations of the measuring probes. Distelhoff et al. (1997) measured blend times defined by $90 \%$ and $95 \%$ at different locations and found that $\tau_{m 90}$ vary up to $27 \%$ and $\tau_{m 95}$ vary up to $21 \%$ among the probes. They also reported that the variation at $\tau_{m} 99$ is much smaller, less than $8 \%$. Most of the reported blend times are the average of the measured data from a small number of probes. Kramers et al. (1953) used two probes, Grenville (1992) used three probes, and Khang and Levenspiel (1976) used 4 probes. Although such averaged blend times can reflect certain characteristics of the mixing process in the tank, they can not completely reveal spatial differences in mixing efficiency caused by the non-uniform flow distribution.

Bakker (2006) presented several ways to measure variations in concentration (for all measures, greater numbers indicate a greater variation with no upper bound):

(a) Coefficient of variation (ratio between the standard deviation and the average concentration): 
where $\sigma=\sqrt{\frac{1}{N} \sum_{i=1}^{N}\left(c_{i}-\bar{c}\right)^{2}}$ and $\bar{c}=\frac{1}{N} \sum_{i=1}^{N} c_{i}$

$$
\operatorname{CoV}=\frac{\sigma_{c}}{\bar{c}}
$$

(b) Ratio between maximum and minimum concentrations

$$
\frac{c_{\max }}{c_{\min }}
$$

(c) Largest deviation between the extremes in concentration and the average concentration:

$$
\Delta_{\max }=\max \left(c_{\max }-\bar{c}, \bar{c}-c_{\min }\right)
$$

Since there is a need to have an absolute measure of uniformity $U$ that is $\leq 1$ with 1 (or $100 \%$ ) indicating perfect uniformity, Bakker (2006) proposed the following uniformity criteria:

(a) Ratio between the minimum and maximum concentrations (bound between 0 and 1)

$$
U_{\min / \max }=\frac{c_{\min }}{c_{\max }}
$$

(b) Based on coefficient of variance $\mathrm{CoV}$ (not bound)

$$
U_{C o V}=1-C o V
$$

(c) Based on largest deviation from the average (not bound; conceptually closer to common experimental techniques)

$$
U_{\Delta}=1-\frac{\Delta_{\max }}{\bar{c}}
$$

All the above-mentioned measures of uniformity indicate perfect uniformity at values of 1 , are not bound between 0 and 1 , and do not take initial conditions into account. In general, it is most useful to be able to predict the time it takes to reduce concentration variations by a certain amount. This can be done by scaling the largest deviation in mass fraction at time $t$ by the largest deviation at time $t=0$.

$$
U_{t}=1-\frac{\Delta_{\max }(t)}{\Delta_{\max }(t=0)}
$$

\subsection{Experimental techniques for determination of the mixing time}

A comprehensive review of available experimental techniques and influence of various parameters has been given by Nere et al. (2003) and Ghotli et al. (2013). Experimental determination of the mixing time involves adding a tracer input (usually a pulse input) at 
some location in the reactor and measuring the tracer concentration as a function of time. The tracer then distributes throughout the vessel, mixes with the fluid in the reactor, until the final uniform concentration is achieved. The mixing time is defined as the time required to achieve a certain degree of uniformity. The tracer used can be a chemical species (inert or reacting), an electrolyte, or a thermal species. The measurement method depends on the type of the tracer and over the past several years, different measurement techniques have been developed (Nere et al., 2003) such as: (i) visual, (ii) conductivity, (iii) thermal, (iv) electrical impedance/resistance tomography, (v) laser-induced fluorescence (LIF), (vi) liquid-crystal thermography, and (vii) computer tomography with coherent light.

In this work the laser-induced fluorescence has been used. The LIF is a spectroscopic method used for studying structure of molecules, detection of selective species and flow visualization and measurements. The species to be examined is excited with a laser. The excited species will after some time, usually in the order of few nanoseconds to microseconds, de-excite and emit light at a wavelength longer than the excitation wavelength. This process can be captured on a camera to assess the mixing characteristics. The mixing time is calculated as the time required for attaining a picture with uniform colour throughout. This technique offers the same advantages as those given by the visual techniques. In addition, the mixing process throughout the tank can be monitored clearly as a function of time. The disadvantage is that the LIF requires a transparent reactor, which is rarely the case on an industrial scale.

\subsection{Mathematical models for prediction of the mixing time}

The rate of mixing of the species often is the controlling factor for the quality of the final product. In general, the mixing process in a turbulent flow in a stirred tank can be divided into three transport processes that each acts at a different range of length scales. At the largest scales, the fluid materials are convected around the reactor at a rate controlled by the mean flow velocity. At the intermediate scales, mixing of the materials is further enhanced by turbulent diffusion due to the turbulent fluctuation of eddies of all sizes. At the smallest scales, molecular diffusion smooths out any remaining concentration gradients. The characteristic mixing times associated with each of these three ranges of scales are different. Nere et al. (2003) and Ghotli et al. (2013) wrote an expanded review of mixing time models and divided them into five categories: (a) semi-empirical correlations based on experimental data, (b) models based on bulk flow, which assume that the process is controlled by the bulk or convective flow, (c) dispersion based models, (d) models that segregate the whole stirred 
vessel into a network of interconnected zones, and (e) CFD models. The characteristics of those types of models were extensively discussed by Nere et al. (2003) and Ghotli et al. (2013). In this work the CFD approach has been adopted and the Smoothed Particle Hydrodynamics method (Nikolic and Frawley, 2015) used for determination of the mixing time. CFD models can be considered as a further advancement of the network of zones models as the local flow structure is resolved using the basic transport equations and the analysis takes into account local convection, dispersion, and its variation throughout the stirred vessel.

\section{Application of the concept of intensity of segregation to crystallisation}

Since the assumption of the ideal mixing conditions produce inaccurate results and the computational expense of CFD models is still prohibitively high, the concept of the intensity of segregation is adopted in this work to take into account the effect of non-ideal mixing. The approach is based on work of Toor (1969). When a chemical reaction takes place in a reactor with turbulent conditions the time-averaged reaction rate depends on the inhomogeneous concentration field, if the reaction is not first order (Toor, 1969). The time-averaged reaction rate for the second-order irreversible and isothermal reaction thus becomes:

$$
\overline{r_{A}}=-k C_{A} C_{B}=-k\left(\overline{C_{A} C_{B}}+\overline{C_{A}^{\prime} C_{B}^{\prime}}\right)
$$

The derivation of this equation depends on the theory developed by Toor (1969) and assumes that with and without reaction the covariances of the reactant fluctuations are the same, and given by:

$$
\overline{C_{A}^{\prime} C_{B}^{\prime}}=I(t) \overline{C_{A 0} C_{B 0}}
$$

Substituting the equation (13) into equation (12) we get:

$$
\overline{r_{A}}=-k\left(\overline{C_{A} C_{B}}-I(t) \overline{C_{A 0} C_{B 0}}\right)=-k\left(\overline{C_{A} C_{B}}-e^{-\frac{t}{\tau_{m}}} \overline{C_{A 0} C_{B 0}}\right)
$$

The kinetics of the crystallisation phenomena such as primary nucleation and growth follow the power law. We can make an analogy to the chemical reaction of the second order and transform the primary nucleation and growth kinetic equations into the following:

$$
\begin{aligned}
& B=k_{b} S^{b}=k_{b}\left(S^{2}\right)^{b / 2} \\
& G=k_{g} S^{g}=k_{g}\left(S^{2}\right)^{g / 2}
\end{aligned}
$$


where $\mathrm{S}$ is a supersaturation rate, $C / C_{e q}$. Following the same logic used by Toor (1969) we can derive the expressions for the modified nucleation and growth rates:

$$
\begin{aligned}
& \bar{B}=\frac{k_{b}}{C_{e q}^{b}}\left(\overline{C^{2}}+\overline{C^{\prime 2}}\right)^{b / 2} \\
& \bar{G}=\frac{k_{g}}{C_{e q}^{g}}\left(\overline{C^{2}}+\overline{C^{\prime 2}}\right)^{g / 2}
\end{aligned}
$$

Finally, applying the equation (13) the modified kinetics expressions are given by:

$$
\begin{aligned}
& \bar{B}=k_{b}^{\prime}\left(\overline{C^{2}}-I(t) \overline{C_{0}^{2}}\right)^{b / 2} \\
& \bar{G}=k_{g}^{\prime}\left(\overline{C^{2}}-I(t) \overline{C_{0}^{2}}\right)^{g / 2}
\end{aligned}
$$

where $k_{b}^{\prime}=\frac{k_{b}}{C_{e q}^{b}}$ and $k_{g}^{\prime}=\frac{k_{g}}{C_{e q}^{g}}$. This way the crystallisation kinetics now includes the effect of non-idealities in the reactor and in particular it is applicable to anti-solvent crystallisations. The shortcomings of this approach are: (a) the models of the phenomena that depend on the local hydrodynamics conditions such as secondary nucleation, aggregation, agglomeration and breakage do not benefit from it, (b) all non-idealities are lumped into a single parameter; thus, it is not possible to identify what is the origin of the non-idealities, and (c) positive and negative fluctuations in concentration are averaged and the effect of local concentration extrema cannot be taken into consideration. 


\section{Materials and methods}

\subsection{Experimental configuration}

The experimental set-up is presented in Fig. 3. The LIF system was purchased from LAVision $\mathrm{GmbH}$ (www.lavision.com) and consists of the continuous wave DPSS laser (10 W, $532 \mathrm{~nm}$ ), Phantom v1211 high-speed CMOS camera (maximal resolution of 1280x800 pixels, 12000 $\mathrm{Hz}$ at full resolution and $48 \mathrm{G}$ RAM), Nicon camera lenses, LIF camera filter for emission wavelengths $>545 \mathrm{~nm}$, light sheet optics and Davis 8 software with the LIF module. The camera rate was set to $100 \mathrm{~Hz}$. The stirred vessel used was KGW Isotherm TRGN-6909 glass reactor of 1 lit with an internal diameter of $100 \mathrm{~mm}$. Baffled configurations used four full height Perspex baffles. The vessel was placed inside a Perspex square tank filled with water to reduce problems associated with refraction by the curved surface. The mixing vessel was mounted on a KGW Isotherm bench top vessel mounting. Perspex baffles were made by 3D printing and presented in Fig. 4. Three different types of impellers from MixerDirect (www.mixerdirect.com) were used and shown in Fig. 5: radial (four $90^{\circ}$ blades), propeller (3 blades) and hydrofoil (4 blades). The impellers were coupled to a stainless steel shaft with an external diameter of $3 / 8$ in. All the impellers had a diameter of 1.5 in and a bottom clearance of $1 / 3$ of the liquid height. The shaft, impellers and baffles were painted in black to avoid reflections. The impeller shaft was driven by a digital 120W CAT R50D overhead stirrer. Tap water at $18-20^{\circ} \mathrm{C}$ was used as a working liquid and the passive scalar was Rhodamine $6 \mathrm{G}$ fluorescence dye. The laser plane was focused using the light sheet optics to obtain approximately $0.5 \mathrm{~mm}$ thick light sheet inside the reactor. The light sheet formed a vertical plane located $0.5 \mathrm{~cm}$ from the shaft to avoid the vortex effect in non-baffled configurations. The camera was positioned perpendicularly to the laser light sheet. Measurements were obtained in the rotational speed range from 300 to 700 and the Reynolds numbers given in Table 1, where the Reynolds number is defined as:

$$
R e=\frac{\rho N D^{2}}{\mu}
$$

where $N$ is the rotational speed in rot/s, $D$ is the impeller diameter $(38.085 \mathrm{~mm}), \rho$ is the density $\left(1000 \mathrm{~kg} / \mathrm{m}^{3}\right)$ and $\mu$ is the dynamic viscosity of the fluid (0.001 Pas). 


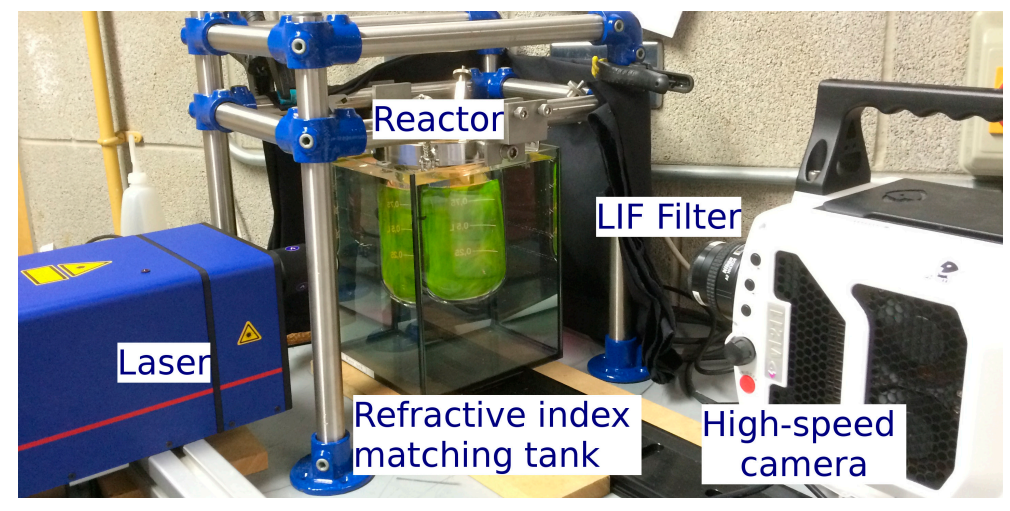

Figure 3. LIF experimental set-up

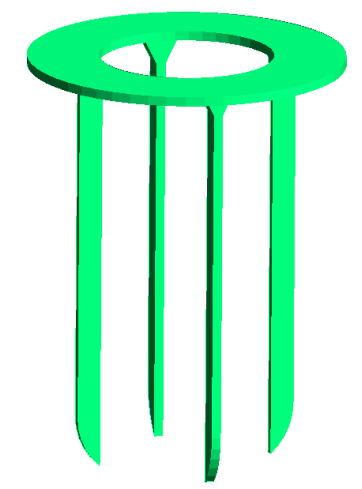

Figure 4. Reactor baffles (width $10 \mathrm{~mm}$, thickness $4 \mathrm{~mm}$ )

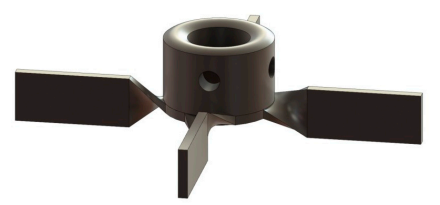

(a) Radial

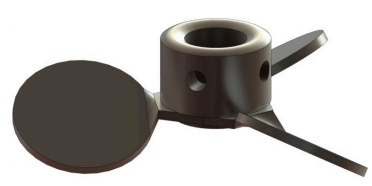

(b) Propeller

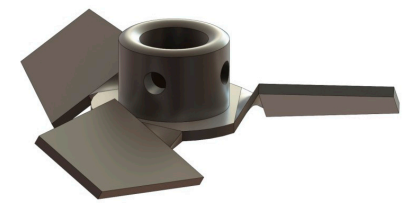

(c) Hydrofoil

Figure 5. MixerDirect lab impellers (1.5in diameter, 3.8in bore)

Table 1. The range of RPMs used and corresponding Re numbers

\begin{tabular}{ccc}
\hline RPM & N, rot/s & Re \\
\hline 300 & 5.000 & 7411 \\
400 & 6.667 & 9882 \\
500 & 8.333 & 12352 \\
700 & 11.667 & 17293
\end{tabular}




\subsection{Experimental procedure}

The experiments are performed by adding a small amount $(0.3 \mathrm{ml})$ of water mixed with the fluorescent dye (Rhodamine 6G) as a pulse input to the reactor. The dye was injected by a syringe at the reactor top, approximately at the middle point between the shaft and a baffle. The concentration of the Rhodamine dye was selected so that the concentrated tracer produces the maximal light intensity (4000 counts) for the given laser power and saturates the camera chip; therefore, the light intensity during the mixing process is always below the saturation limit. The tracer is then in-homogeneously being distributed in the rest of the fluid. The fluorescence signal is proportional to the local dye concentration, that is the light intensity of the recorded images is directly proportional to the local concentration. This requires calibration of the system for several known concentrations which establishes a dependency of the concentration on the intensity of the light (typically linear, as shown in Fig. 6). Since the camera's dark current (dark image) and surrounding light (background image) add an offset to the signal from the actual experiment, in order to extract the pure LIF signal these offsets must be subtracted. Also, all laser beams exhibit a cross-sectional intensity distribution, which is compressed to a profile perpendicular to the beam axis when being formed to a sheet. The inhomogeneities will decrease the accuracy of the results if a uniform intensity distribution is assumed for evaluation and these effects must be corrected by taking a sheet image which contains information on both the laser's spatial inhomogeneity and transmission of the detection system. The experimental procedure is presented in Fig. 7 and consists of the following steps: (a) recording of the raw images (the light intensity), (b) subtraction of a background noise, (c) sheet-correction, (d) masking out the region of interest, (e) concentration calculation (using pre-recorded calibration, as in Fig. 6), and (f) extraction of the statistical data.

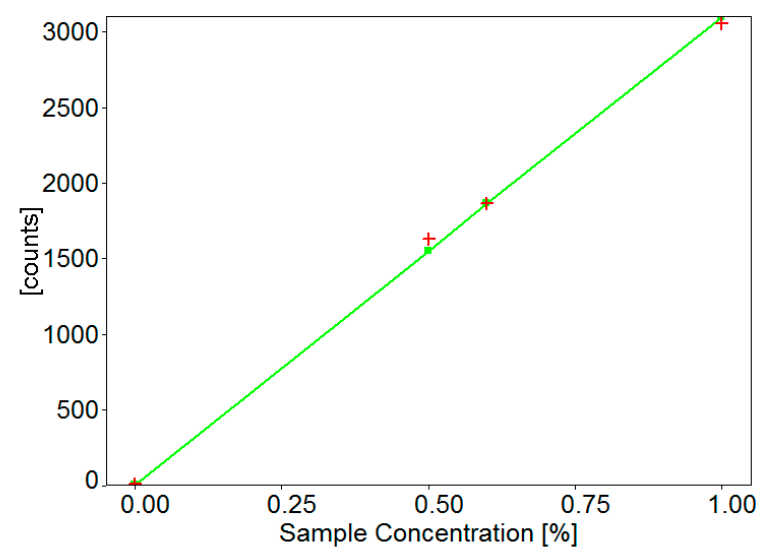

Figure 6. LIF concentration calibration plot: calibration points (red), correlation (green) 


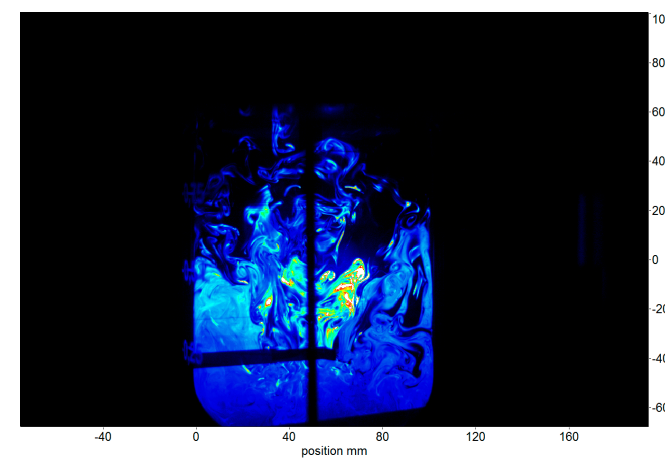

(a) Original image

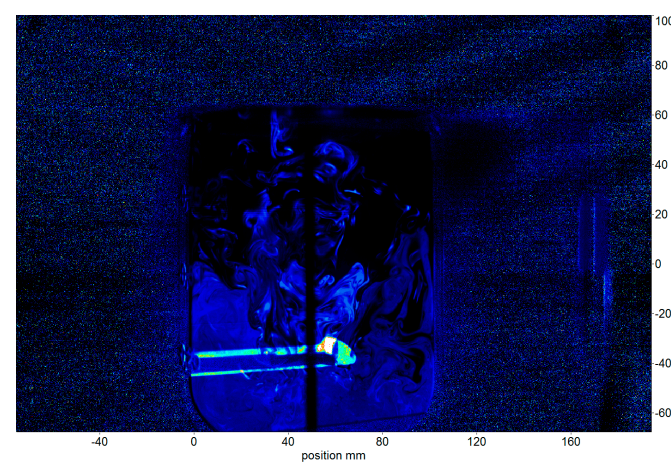

(c) Sheet correction

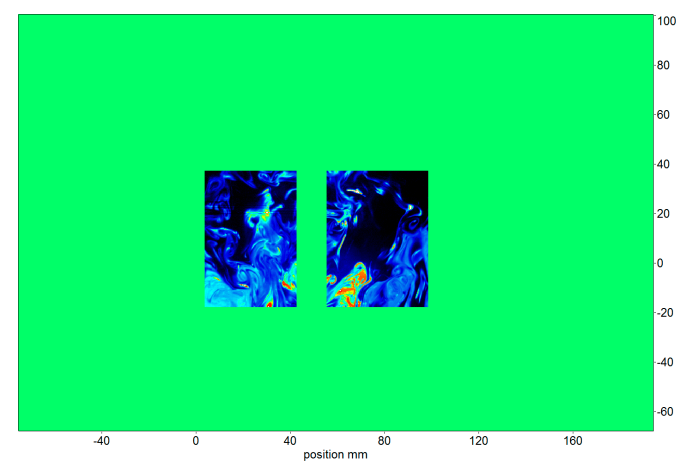

(e) Calculation of the concentration in a smaller area to avoid zones with image granularity, the vortex around the shaft and reflection rays in non-baffled configurations, etc.

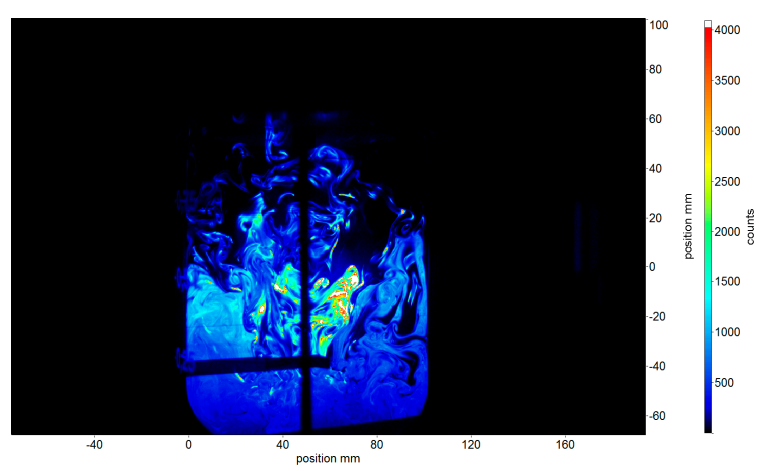

(b) Subtraction of the background noise

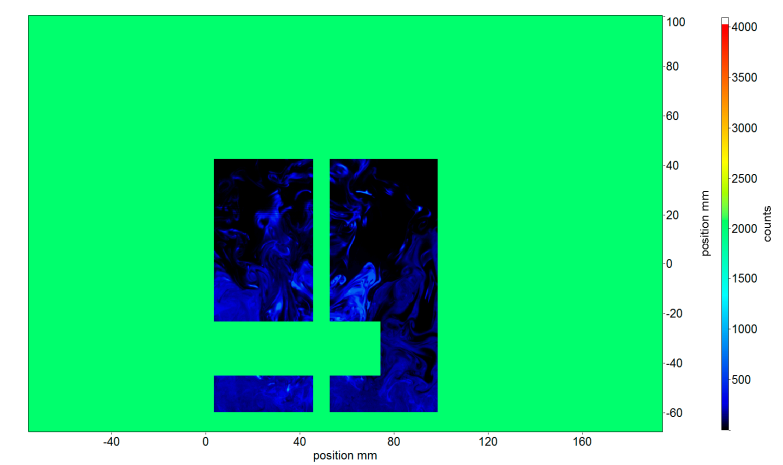

(d) Masking out the region of interest

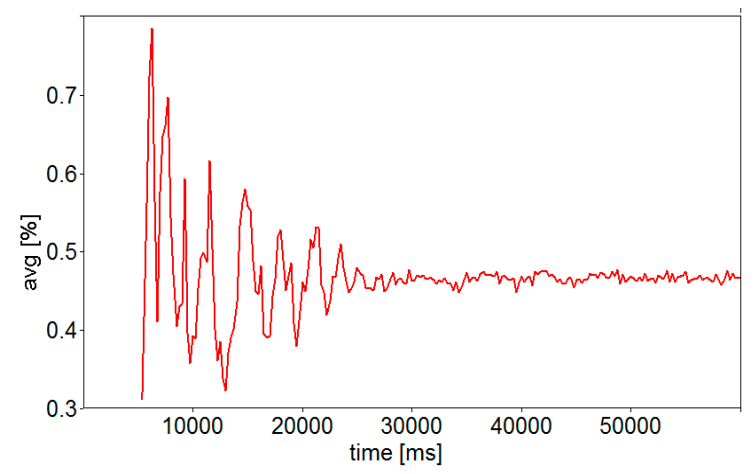

(f) Extraction of statistical data

(i.e. average, stdev, $\min / \max$ etc.)

Figure 7. Processing of the raw LIF experimental images 


\section{Results and discussion}

\subsection{Experimental results}

The experimental set was divided in two groups: non-baffled and baffled configurations, each containing 12 experimental points (3 different impeller types and 4 rotational speeds: 300 , 400, 500 and 700). The measurements were repeated three times for every experimental point. For the points where the results in three repetitions significantly differed the measurements were repeated additional three times (i.e. baffled configurations for hydrofoil impellers with 300 and $700 \mathrm{rot} / \mathrm{min}$ ).

Early experiments were performed using a narrow laser sheet and non-painted baffles, shaft and impellers. The reasons for using the narrow sheet were better utilisation of the laser energy and to avoid reflections from the liquid surface at the top of the reactor and from the impeller blades. However, the processing of the raw images revealed the following problems: (a) the concentration measurements were affected by the light reflected by the baffles, (b) the zones around impellers and at the top of the reactor that in general experience the highest degree of concentration fluctuations could not be monitored. Therefore, all parts in the reactor were painted in black and the larger laser sheet was used. A typical experiment is presented in Video 1 (using 1.5 in radial impellers and $150 \mathrm{rpm}$, with baffles). The most of the problems were resolved except that the very top and the very bottom parts of the reactor had to be masked out because the water surface at the reactor top acts as a mirror and reflects the laser light while the curvature at the bottom obstructs the optical path and also creates reflections. The concentration measurements in those regions could not be performed but they can be visually monitored for the existence of unmixed fluid and the resulting mixing time adjusted. This was particularly pronounced at the low impeller speeds where slowly moving tracer "clouds" were visually observed but could not be recorded by the system. This phenomena can be noticed at the end of Video 1 where the fluid becomes fully mixed everywhere except at the reactor top where the mixing conditions are rather poor.

Other sources of the problems frequently causing the problems with concentration readings are presented in Fig. 8. In configurations without baffles, at high rpm the rotational movement of the fluid generates a vortex around the stirrer shaft. The vortex produces the rays of light (reflections of the laser sheet from the vortex outer surface) which cause the peaks in the measured concentration (as illustrated in Fig. 9) and have to be filtered out from the results. An irregular surface of the reactor wall also generates rays which can be 
automatically filtered to a certain degree during the sheet correction phase. In addition, image granularity was observed in the regions with the low laser sheet intensity (the top and bottom of the laser sheet) and these areas had to be excluded (masked out). Occasionally, the LIF filter has to be cleaned to remove the dust particles and hair stuck onto its surface that can cause false extrema.

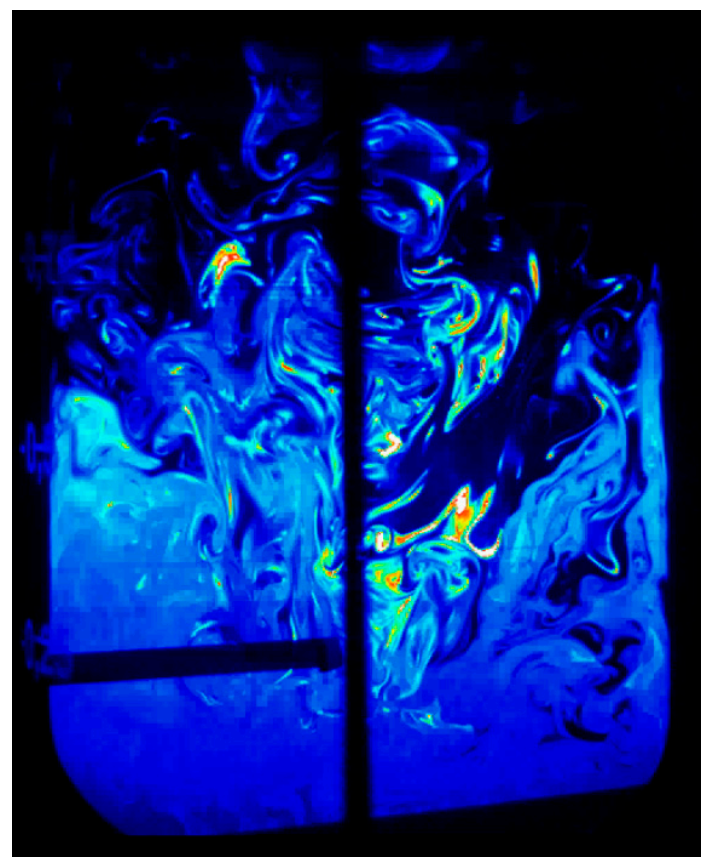

Video 1. The typical LIF experiment
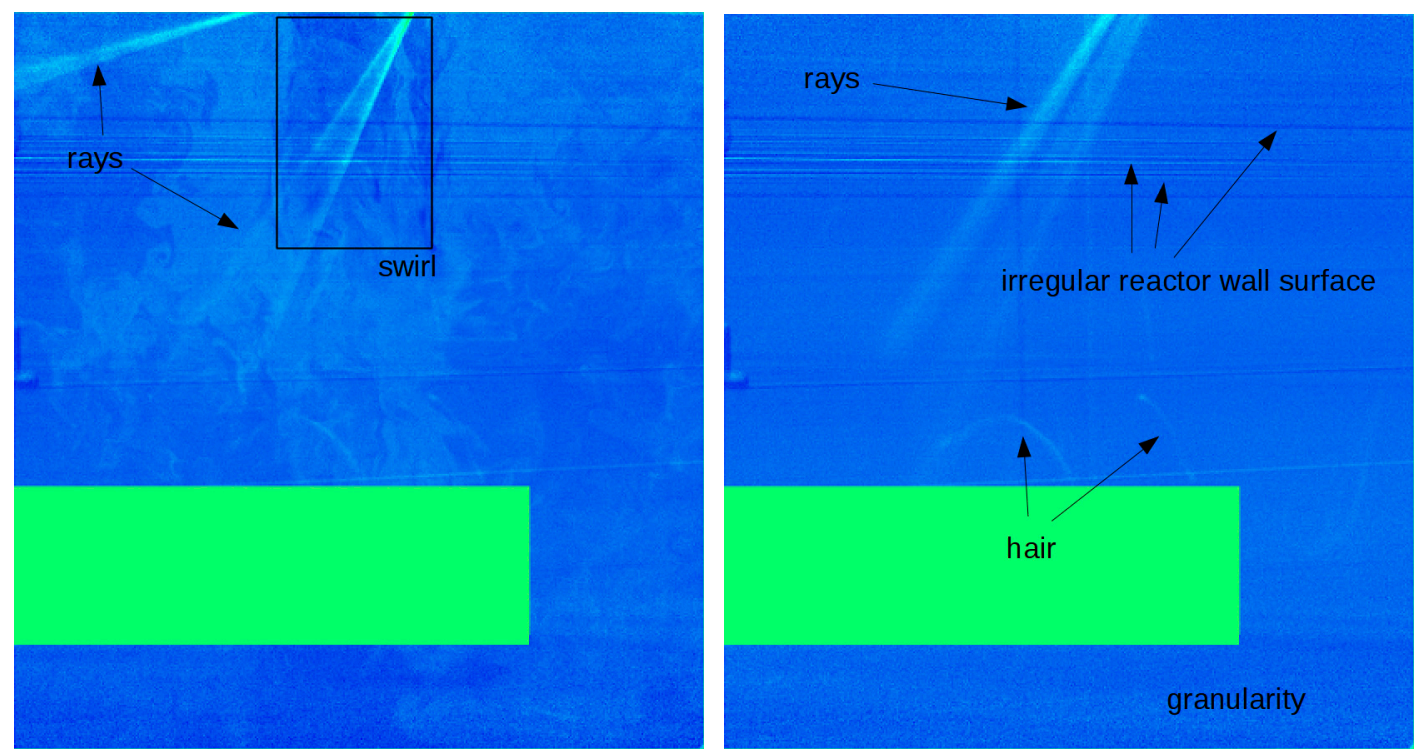

Figure 8. Causes for improper concentration calculations - a vortex and rays around the shaft in non-baffled configurations, granularity in the regions with low laser light intensity, rays because of the irregular reactor surface, hair on the LIF filter 


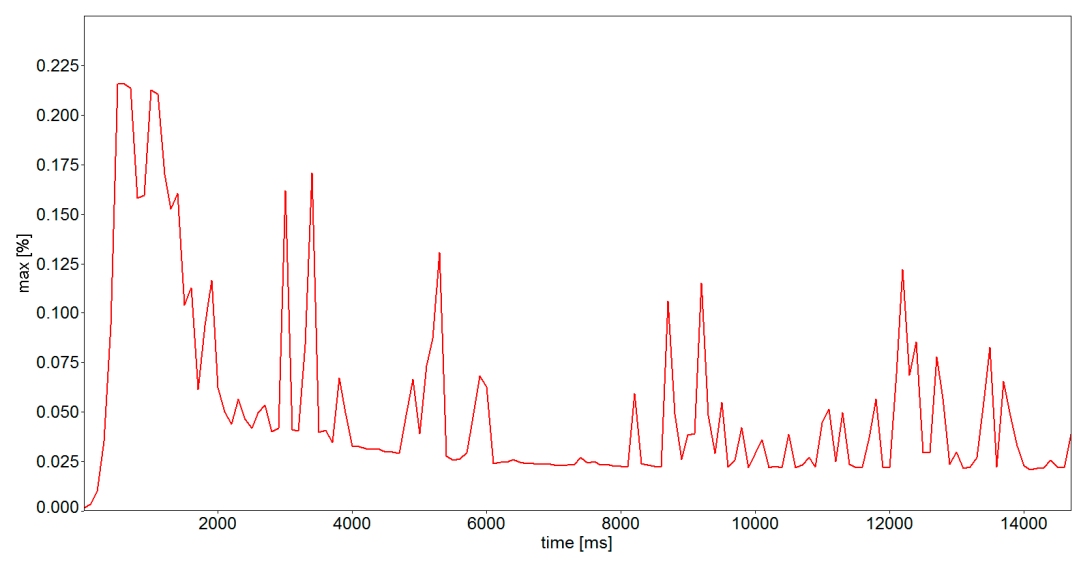

Figure 9. Concentration peaks caused by the rays produced by the vortex

In this work the $U_{t}$ uniformity criterion was applied to determination of mixing time. The main reason is that the fluctuations of the average concentration in some cases were too high to reliably detect the points where the concentrations reached 95 or $99 \%$ of the final concentration. Therefore, it was difficult to consistently apply the $c / c_{\infty}$ criterion. The $U_{\min / \max }$ and $U_{\Delta}$ criteria also produced unacceptably high fluctuations, again due to the concentration oscillations making the difference between $c_{\min }$ and $c_{\max }$ highly oscillating as well (as presented in Fig. 10). The most probable reasons for the occurrence of concentration oscillations are the non-uniform energy of the light produced by the laser and possible reflections. On the other hand, the $U_{C O V}$ criterion never really reaches one since a certain degree of standard deviation is always present.

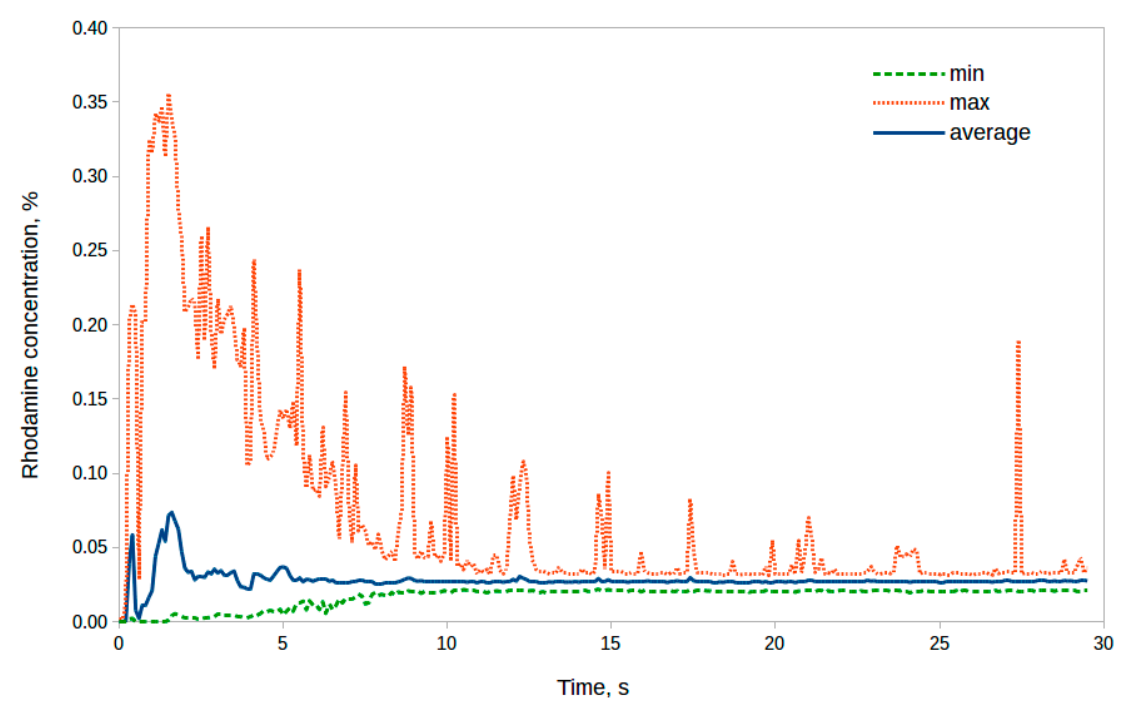

Figure 10. Fluctuations of the maximal concentration; standard deviation never reaches zero since the $c_{\max } c_{\min }$ difference never disappears completely 
The experimental results for the configurations with and without baffles are given in Fig. 11 ( $\tau_{m}$ for $U_{t}=95 \%$ ), Fig. 12 ( $\tau_{m}$ for $U_{t}=99 \%$ ), Fig. 13. (non-dimensional $N \tau_{m}$ for $U_{t}=95 \%$ ), and Fig. 14 (non-dimensional $N \tau_{m}$ for $U_{t}=99 \%$ ). The plots include all experimental points (given by " $x$ " marks) and error bars equal to the standard deviation of the result set.
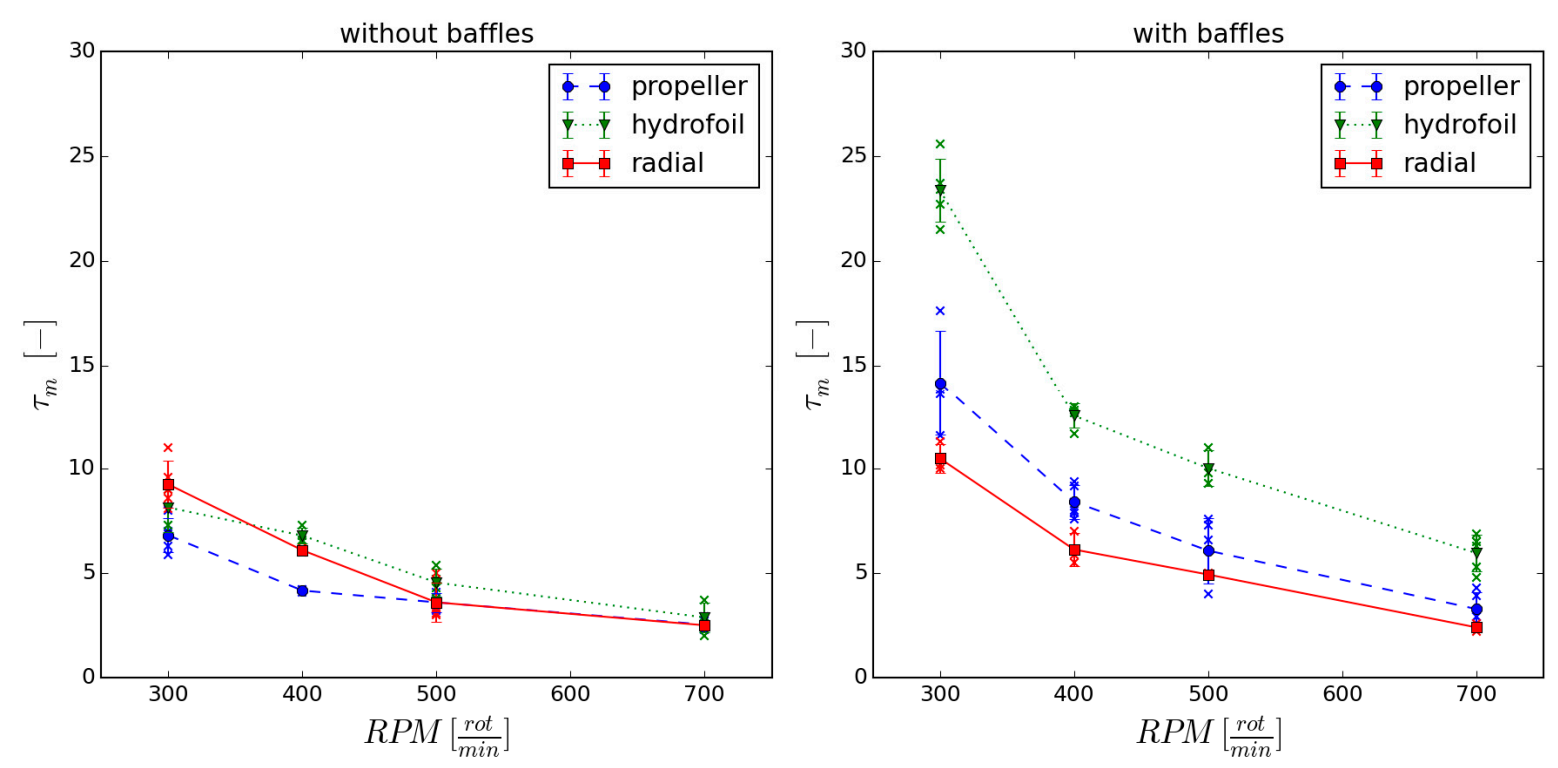

Figure 11. $\tau_{m}$ for $U_{t}=95 \%$ : without baffles (left), with baffles (right), error bars equal to the standard deviation of the result set
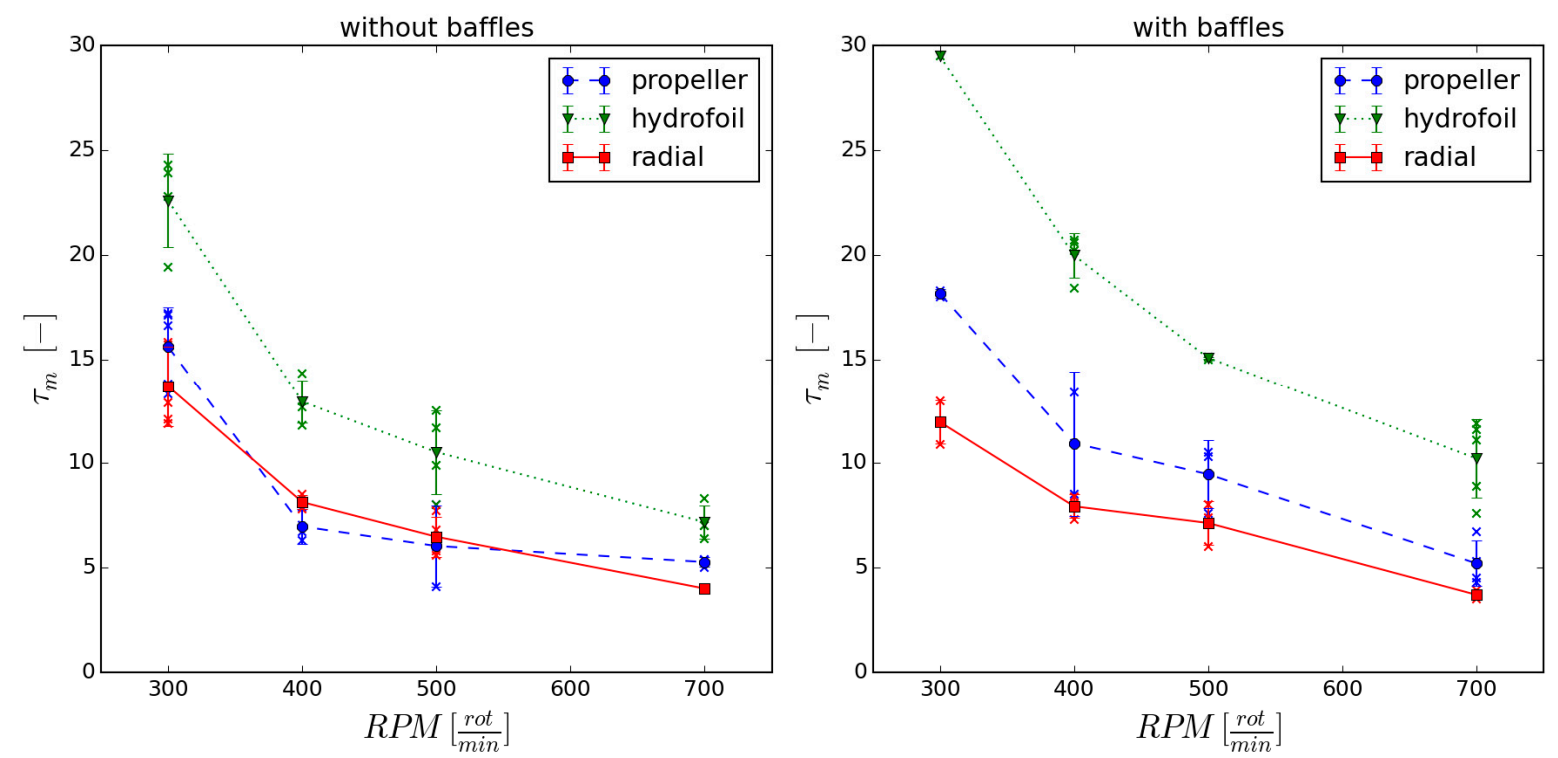

Figure 12. $\tau_{m}$ for $U_{t}=99 \%$ : without baffles (left), with baffles (right), error bars equal to the standard deviation of the result set 

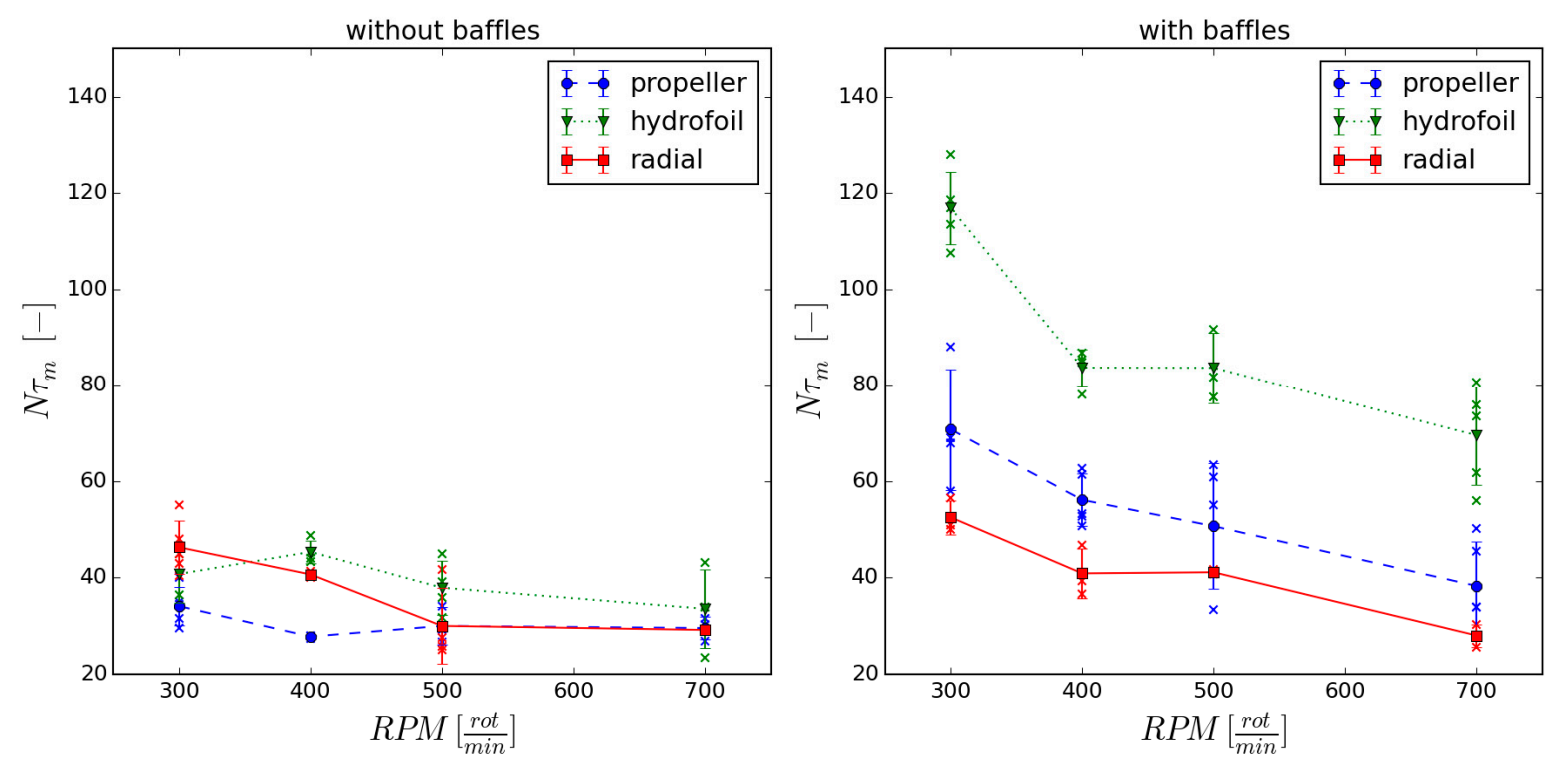

Figure 13. $N \tau_{m}$ for $U_{t}=95 \%$ : without baffles (left), with baffles (right), error bars equal to the standard deviation of the result set
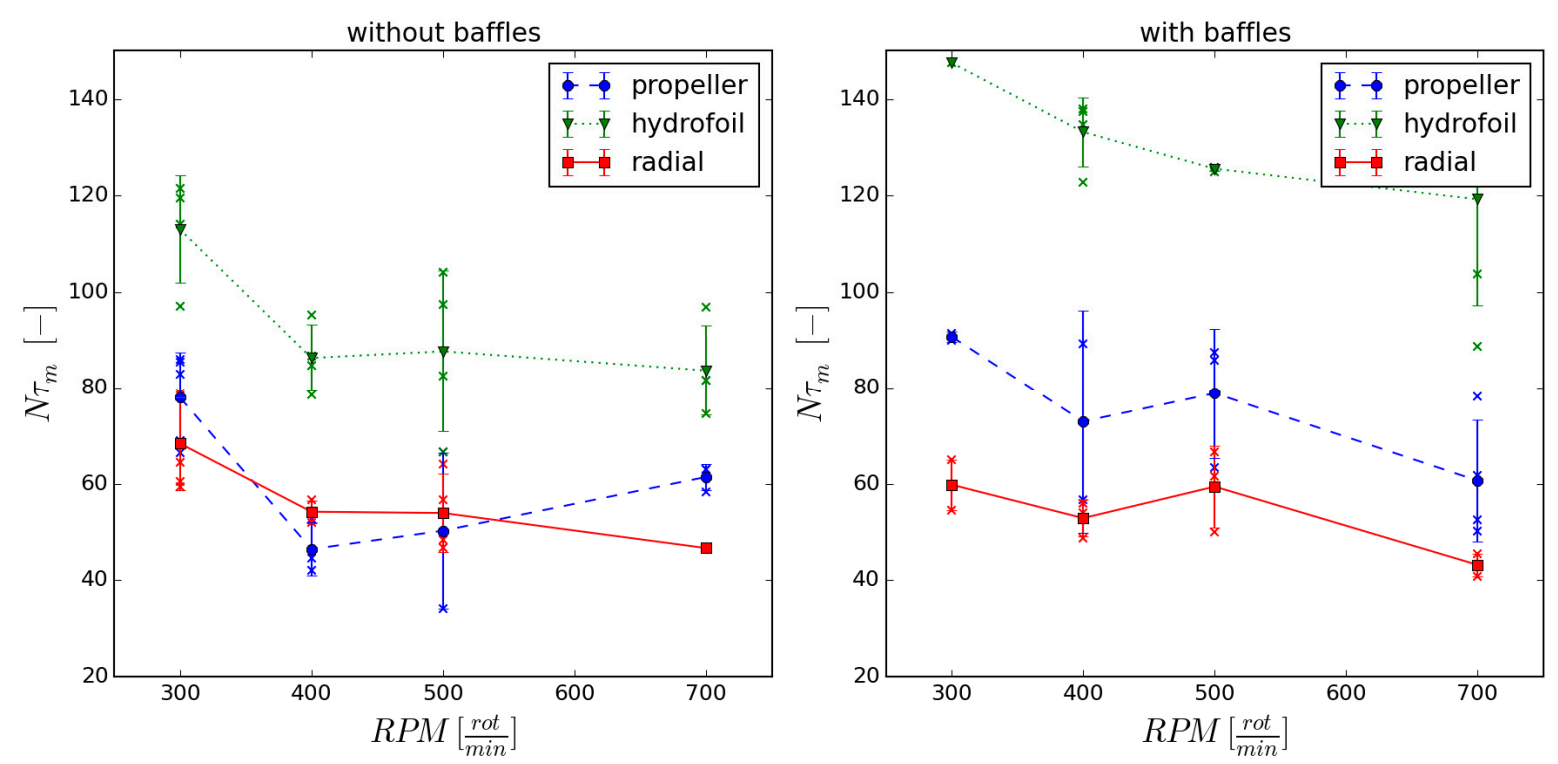

Figure 14. $N \tau_{m}$ for $U_{t}=99 \%$ : without baffles (left), with baffles (right), error bars equal to the standard deviation of the result set 


\subsubsection{Non-baffled configurations}

It could be observed that the mixing time is significantly shorter for non-baffled configurations which is against the fact that baffles increase the pumping power and improve the mixing performance. This can be explained by the fact that configurations without baffles produce a strong rotational movement of the fluid causing the lifting of the liquid level and appearance of a void space and a vortex around the stirrer shaft. The mass transfer within the vortex is basically diffusional and very slow. In addition, the vortex produces light reflections and rays which can be observed in Fig. 15 which is particularly pronounced at higher rpms. To overcome this issue, the region around the shaft had to be masked out and the concentration is not measured there. Consequently, the whole phenomena is completely missed and the measured mixing times are misleading. Therefore, the mixing times for nonbaffled configurations in figures 11 to 14 are incorrect. The reason for this is that after a relatively quick internal homogenization two segregated zones clearly form: a small volume around the shaft between the stirrer and reactor top and the rest of vessel volume. This finding is in agreement with the literature since the existence of the two segregated zones was also reported by Busciglio et al. (2012). To prove that two segregated zones in the reactor without baffles really form, two additional experiments have been performed: (1) the tracer was slowly injected into the vortex around impellers shaft (Video 2), and (2) the tracer was injected close to the reactors wall, far away from the vortex (Video 3). In the experiment (1), the tracer stays trapped within the vortex for a long time and after moving down and reaching the impellers it gets slowly released into the rest of the fluid. In the experiment (2), in the beginning the fluid around the shaft stays free of the tracer for a long time; then a part of the concentrated tracer enters the vortex and gets trapped within; finally, as in the experiment (1) the tracer gets slowly released into the rest of the fluid. The precise measurement of the mixing time in non-baffled configurations therefore was difficult and limited to visual observations only. The highest degree of difficulties were observed in configurations with high rpms due to the reflection rays and the large vortex around the shaft producing the concentration fluctuations with a large magnitude. 

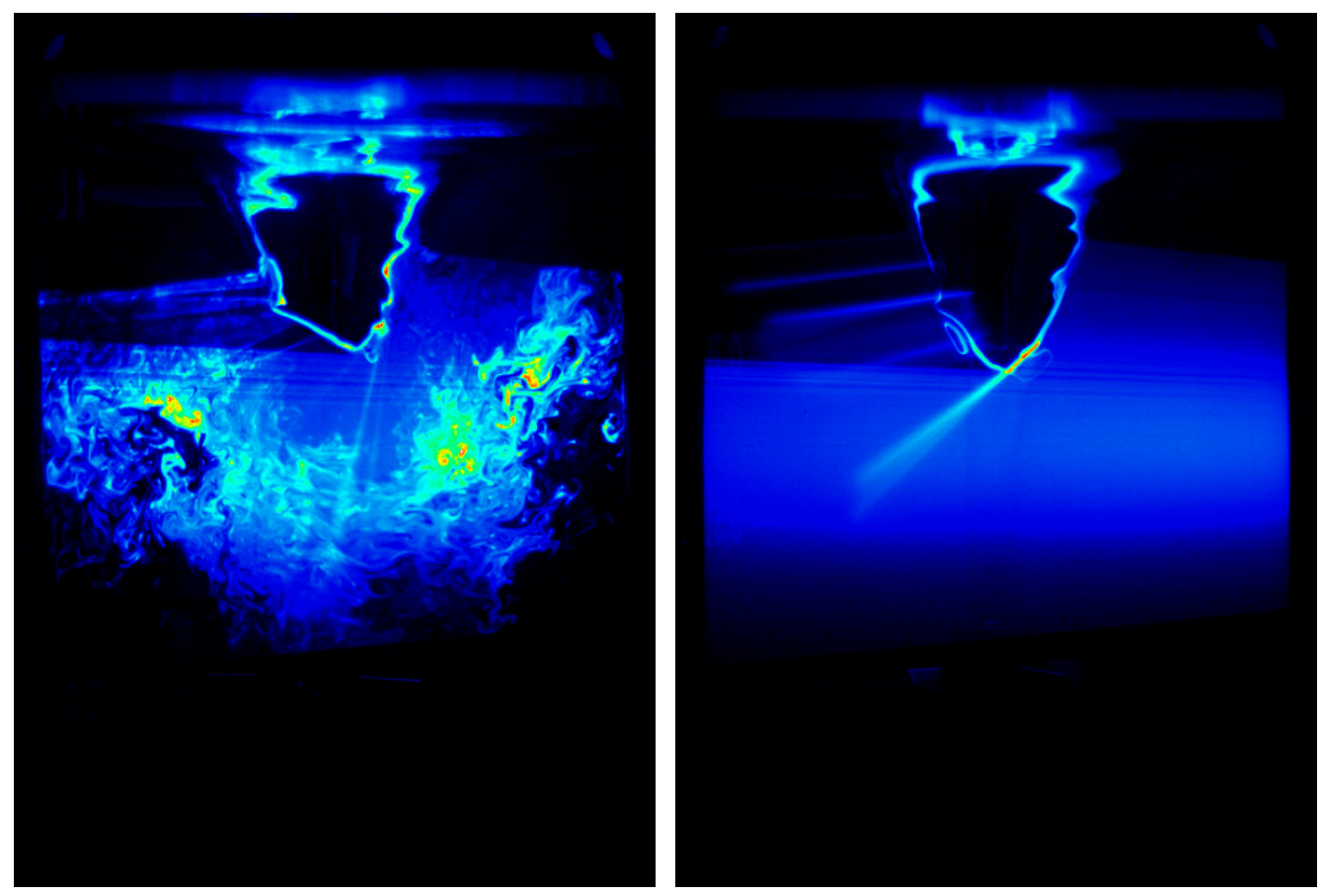

Figure 15. Non-baffled configurations - the rays and vortex around the stirrer shaft

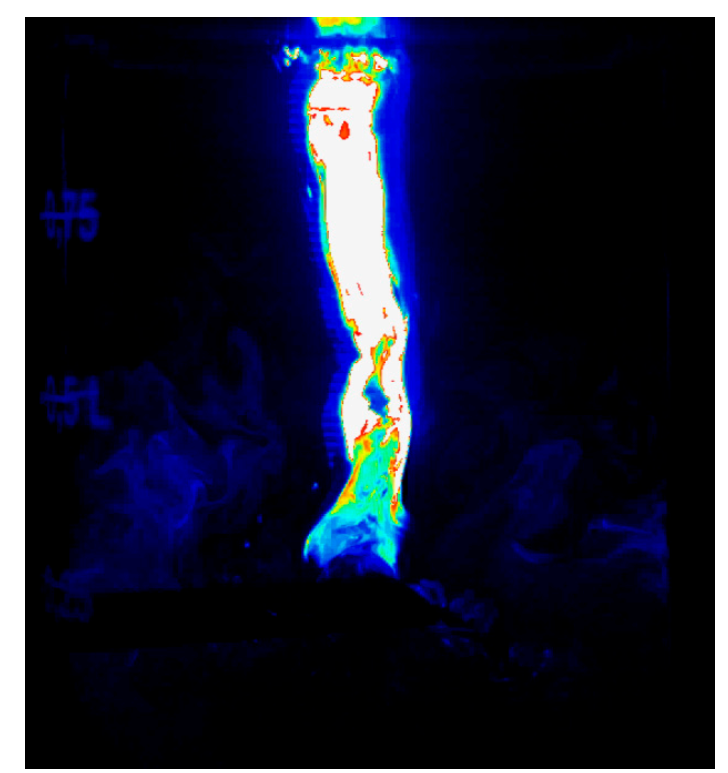

Video 2. Non-baffled configurations - effects of injection of the tracer in the vortex around the stirrer shaft (the tracer being trapped within the vortex) 


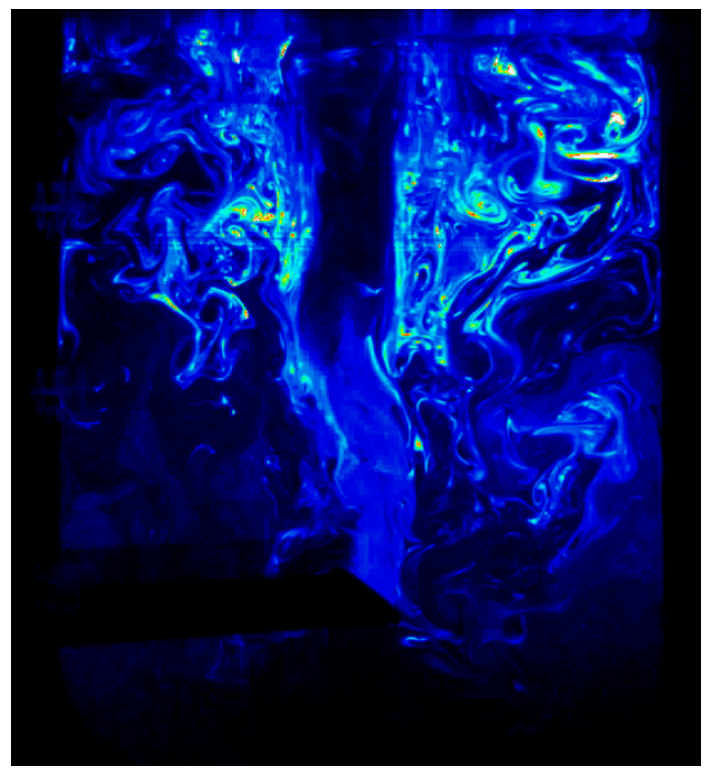

Video 3. Non-baffled configurations - effects of injection of the tracer close to the reactor wall (no tracer in the vortex around the shaft)

\subsubsection{Baffled configurations}

From the results for baffled configurations it can be concluded that the radial flow impellers give the shortest mixing times. This unusual finding can be explained by the fact that they produce much higher shear due to their very small height of only $4 \mathrm{~mm}$. An additional factor could be a design of MixerDirect lab impellers which differ from the design of the industrial ones (basically they are made by bending the thick straight blades by hand), as illustrated in Fig. 5. The results also show the existence of dead zones in the reactor at the low impeller speeds where slowly moving tracer "clouds" were observed at the top of the reactor and behind the baffles close to the reactor wall. As already pointed out in the previous section these phenomena can be observed at the end of Video 1. Therefore, the LIF technique can be a valuable tool for troubleshooting/de-bottlenecking and detection of dead zones. From the Fig. 13 and 14 we can observe that dimensionless mixing time $\left(N \tau_{m}\right)$ is not constant, as previously reported in the literature by many authors. The reasons are that the flow is not fully turbulent for low rpms. However, if only the points with the turbulent conditions are taken into the account (with $\operatorname{Re}>10000, \mathrm{rpm}>=500$ ) only the minimal differences in the dimensionless mixing time exist, which is in accordance with the previous findings.

\subsection{Simulation results}

The mixing time simulations were performed using the SPH model presented in Nikolic and Frawley (2015) extended with the standard k- $\varepsilon$ turbulence model. The reactor geometry in the 
simulation was identical to the experimental configuration. Only the simulations for the 1.5 in radial flow impellers were carried out. The comparison between the experimental data (LIF) and simulation results (SPH) is given in figures 16 and 17. The corresponding percentage deviations are given in Table 2.

The mixing times from the simulations are significantly shorter than the experimental values for $U_{t}=95 \%$, particularly at low rpms where the system is not fully turbulent yet. This can be attributed to the fact that k- $\varepsilon$ model was developed and tested for fully turbulent conditions which is not the case for lower rpms. Therefore, the turbulent diffusivity is over-predicted and consequently the mixing times are shorter. In addition, as it is previously reported in the literature, the standard k- $\varepsilon$ model performs poorly for rotating flows such as stirred tanks.

On the other hand, the mixing times from the simulations for $U_{t}=99 \%$ are significantly longer. Again, this is more pronounced at low rpms. The main reason for this is that the SPH model under-predicts the size of two main circulation loops in the reactor as already reported in Nikolic and Frawley (2015) and illustrated in Fig. 18. The figure shows the SPH model prediction of the height of two main circulation loops in a stirred tank from Hartmann et al. (2004). The data are obtained for the axial plane located $15^{\circ}$ behind impellers. The height of the upper circulation loop in the SPH model is approximately $25 \%$ shorter than the height obtained by the LDA experiments. The most probable cause is the use of the weakly compressible fluid flow model in SPH which applies artificial, physically non-existent forces between fluid particles to preserve approximately uniform density. These forces dampen the fluid velocity (particularly close to the reactor walls). Thus, the largest scales where the fluid is convected around the reactor by the mean flow velocity are not well predicted and consequently, a small amount of the tracer gets trapped at the top of the reactor close to the baffles and slows down the overall mixing process. 

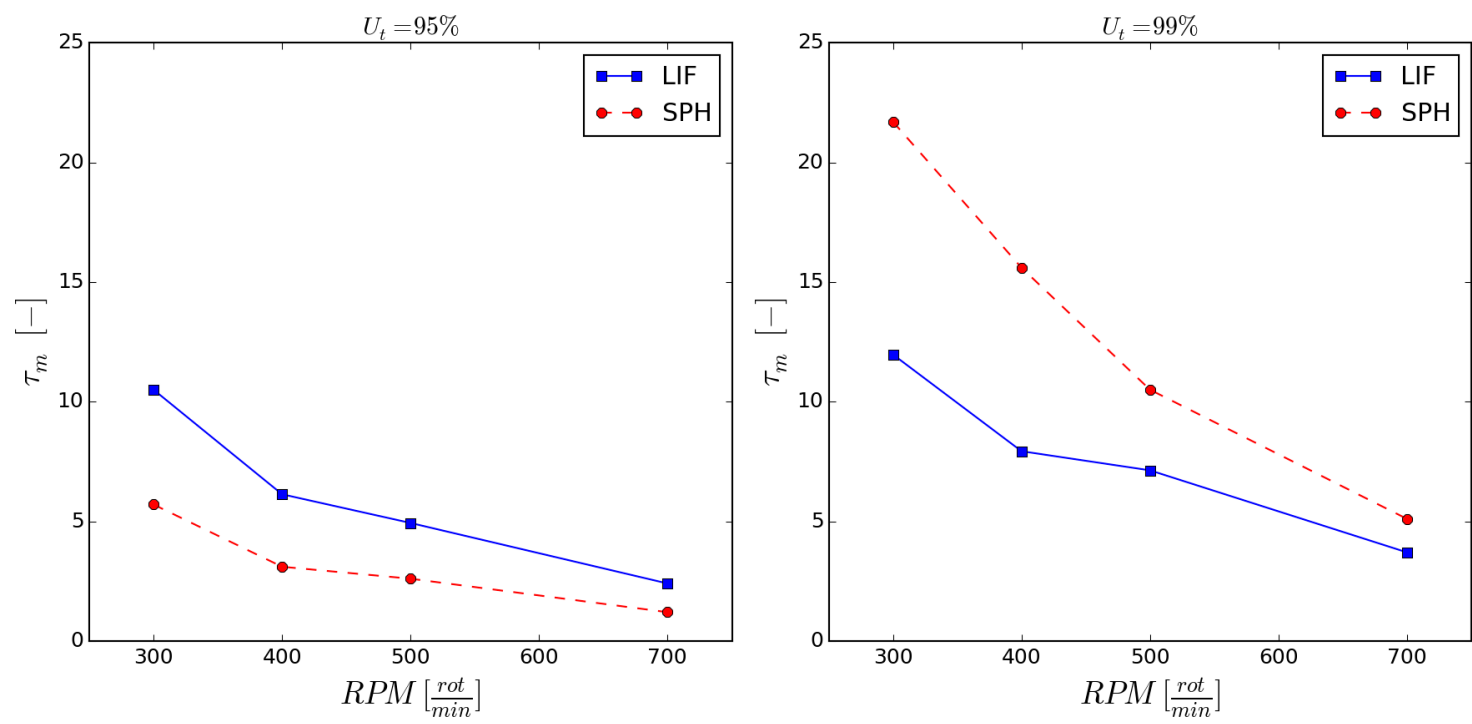

Figure 16. Comparison of $\tau_{m}$ between the experimental data (LIF) and simulation results (SPH) for radial impellers
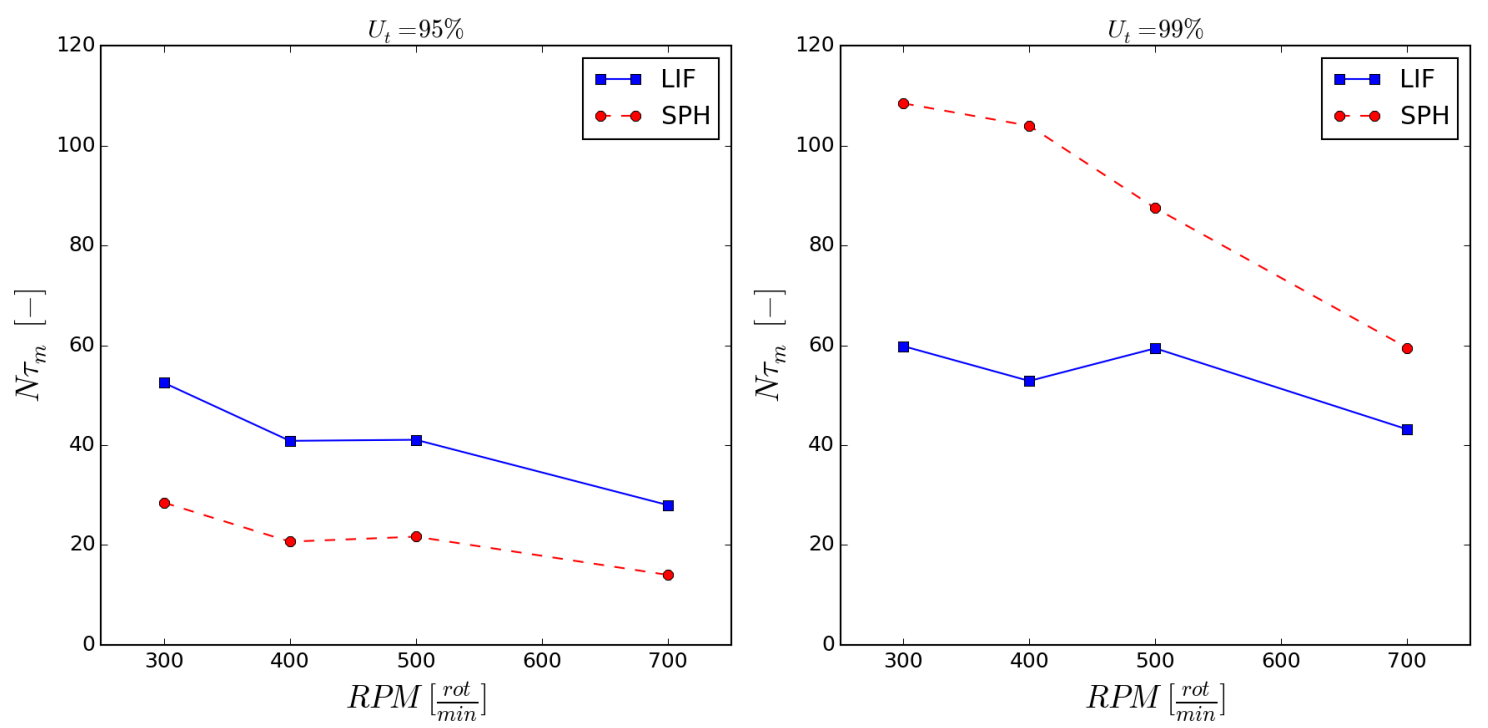

Figure 17. Comparison of $N \tau_{m}$ between the experimental data (LIF) and simulation results (SPH) for radial impellers

Table 2. Percentage deviation in $\tau_{m}$ between the experimental data and simulation results

\begin{tabular}{|c|c|c|c|c|}
\hline 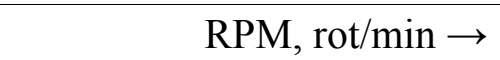 & 300 & 400 & 500 & 700 \\
\hline & 45.7 & 49.4 & 47.3 & 50 \\
\hline$\tau_{\mathrm{m} 99, \mathrm{SPH}} \mid / \tau_{\mathrm{m} 99, \mathrm{LIF}}, \%$ & 81.3 & 96.7 & 47.3 & 37.8 \\
\hline
\end{tabular}




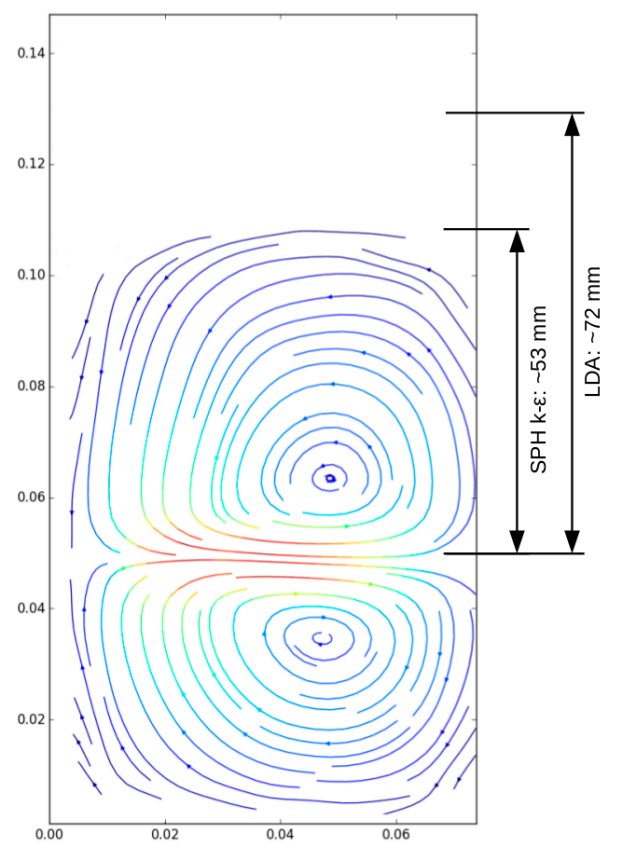

Figure 18. Prediction of two main circulation loops in a stirred tank: SPH simulation results vs. LDA experimental data (Hartmann et al., 2004)

\section{Conclusions and future work}

In this work, a new approach to taking into consideration the effect of non-ideal mixing on crystallisation processes has been proposed which requires less computational resources than full CFD simulations. The starting point is the Danckwerts concept of the intensity of segregation, and an analogy between its application to chemical reactions and the kinetics of the crystallisation phenomena has been made. The obtained modified kinetics expressions now incorporate the effect of non-idealities in the stirred reactor. This way, based on the experimental measurements of the mixing time using the LIF technique the computational requirements can be significantly decreased. The experimental measurements of the mixing time using the LIF technique for configurations with and without baffles for three types of impellers and four different rotational speeds have been presented. The non-physical results such as shorter mixing times in non-baffled configurations have been visualised and explained. The precise measurements in those cases has been shown as difficult, particularly for the higher rpms due to the concentration fluctuations caused by the reflection rays and the large vortex around the shaft. The experimental data has been compared to the preliminary simulation results obtained from the SPH method and the standard k- $\varepsilon$ turbulence model with the modest success. 
The future work will concentrate on application of improved definitions of the mixing quality, a detailed experimental campaign to obtain the effect of various design and operating parameters on the mixing efficiency, fine tuning of the SPH model using the data from the Particle Image Velocimetry experiments and implementation of additional turbulence models.

Acknowledgements: This research has been conducted as part of the Synthesis and Solid State Pharmaceutical Centre (SSPC) and funded by Science Foundation Ireland (SFI).

\section{Nomenclature}

$B$ - birth rate, $\# /\left(\mathrm{m} \mathrm{m}^{3} \mathrm{~s}\right)$

$b$ - exponent in the primary nucleation rate equation

$c, C_{A}, c_{i}-$ concentration, $\mathrm{mol} / \mathrm{m}^{3}$

$\mathrm{c}_{\infty}$ - tracer concentration after infinite time; the final mixing concentration, $\mathrm{mol} / \mathrm{m}^{3}$

$C_{A}^{\prime}$ - concentration fluctuation about the mean, $\mathrm{mol} / \mathrm{m}^{3}$

$c_{p}$ - heat capacity, $\mathrm{J} /(\mathrm{kg} . \mathrm{K})$

$C_{e q}-$ solubility concentration, $\mathrm{mol} / \mathrm{m}^{3}$

$\Delta C$ - supersaturation, $\mathrm{mol} / \mathrm{m}^{3}$

$D$ - impeller diameter, $\mathrm{m}$

$g$ - exponent in the growth rate equation

$G-$ growth rate, $\mathrm{m} / \mathrm{s}$

$I$ - intensity of segregation, -

$k_{b}, k_{b}^{\prime}-$ constant in the primary nucleation rate equation

$k_{g}, k_{g}^{\prime}$ - constant in the growth rate equation

$N$ - rotational speed, rot/s

$p$ - pressure, $\mathrm{Pa}$

$r_{A}-$ reaction rate

$R e-$ Reynolds number, -

rpm, $R P M$ - rotational speed, rot $/ \mathrm{min}$

$S$ - supersaturation rate $\left(\mathrm{C} / \mathrm{C}_{\mathrm{eq}}\right)$, rot $/ \mathrm{min}$

$T$ - temperature, $\mathrm{K}$

$u$ - velocity, $\mathrm{m} / \mathrm{s}$

$v_{A}, v_{B}$ - volume of components $\mathrm{A} / \mathrm{B}, \mathrm{m}^{3}$

$V$ - reactor volume, $\mathrm{m}^{3}$

Greek symbols

$\lambda$ - thermal conductivity, $\mathrm{W} /(\mathrm{m} . \mathrm{K})$

$\mu$ - viscosity, Pa.s

$\rho$-density, $\mathrm{kg} / \mathrm{m}^{3}$

$\tau_{\mathrm{m}}$ - Mixing time, $\mathrm{s}$

$\mathrm{N} \tau_{\mathrm{m}}$ - Dimensionless mixing time (total impeller revolutions), - 


\section{References}

Bakker A. (2006), Blend Times in Stirred Tanks: Reacting Flows - Lecture 9, available at http://www.bakker.org/dartmouth06/engs 199/09-blend.pdf (accessed 11 October 2015).

Busciglio A., Grisafi F., Ippolito F., Scargiali F., Brucato A. (2012), Mixing time in unbaffled stirred tanks, $14^{\text {th }}$ European conference on mixing, Warszawa, 10-13 September 2012.

Danckwerts P.V. (1952), The definition and measurement of some characteristics of mixtures, Appl. Sci. Res. A3:279-296.

Danckwerts P.V. (1958), The effect of incomplete mixing on homogeneous reactions. Chem. Eng. Sci. 8:93-102. doi:10.1016/0009-2509(58)80040-8.

Distelhoff M.F.W., Marquis A.J., Nouri J.M., Whitelaw J.H. (1997), Scalar mixing measurements in batch operated stirred tanks. Can. J. Chem. Eng. 75:641-652. doi:10.1002/cjce.5450750401

Ghotli A.R., Raman A.A.A., Ibrahim S., Baroutian S. (2013). Liquid-liquid mixing in stirred vessels: A review. Chem. Eng. Commun. 200:595-627. doi:10.1080/00986445.2014.938805.

Grenville R.K. (1992), Blending of Viscous Newtonian and Pseudo-Plastic Fluids, Ph.D. dissertation, Cranfield Institute of Technology, Cranfield, Bedfordshire, England.

Hartmann H., Derksen J.J., Montavon C., Pearson J., Hamill I.S., van den Akker H.E.A. (2004), Assessment of large eddy and RANS stirred tank simulations by means of LDA, Chem. Eng. Sci. 59, 2419-2432. doi:10.1016/j.ces.2004.01.065.

Ingham J., Dunn I. J., Heinzle E., Prenosil J.E., Snape J.B. (2007), Chemical Engineering Dynamics, WILEY-VCH

Khang S.J., Levenspiel O. (1976), New Scale Up and Design Method for Stirrer Agitated Batch Mixing Vessels. Chem. Eng. Sci. 31:569. doi:10.1016/0009-2509(76)80020-6.

Kramers H., Baars G.M., Knoll W.H. (1953), A comparative study on the rate of mixing in stirred tanks. Chem. Eng. Sci. 2:35-42. doi:10.1016/0009-2509(53)80006-0.

Kukukova A., Aubin J., Kresta S. (2009), A new definition of mixing and segregation: Three dimensions of a key process variable, Chem. Eng. Res. Des. 87:633-647. doi:10.1016/j.cherd.2009.01.001.

Nere N.K., Patwardhan A.W., Joshi J.B. (2003). Liquid-Phase Mixing in Stirred Vessels: Turbulent Flow Regime. Ind. Eng. Chem. Res. 42, 2661-2698. doi:10.1021/ie0206397.

Nikolic D.D., Frawley J.P. (2015), Application of the Lagrangian Meshfree Approach to Modelling of Batch Crystallisation: Part I - Modelling of Stirred Tank Hydrodynamics, Chem. Engi. Sci., Available online 28 September 2015. doi:10.1016/j.ces.2015.08.052.

Paul L.P., Atieno-Obeng V.A., Kresta S.M. (2004), Handbook of Industrial Mixing, WileyInterscience.

Ranade V.V., Bourne J.R., Joshi J.B. (1991), Fluid mechanics and blending in agitated tanks. Chem. Eng. Sci. 46:1883-1893. doi:10.1016/0009-2509(91)80150-W.

Toor H.L. (1969), Turbulent mixing of two species with and without chemical reactions, Ind. Eng. Chem. Fund. 8:646-655. doi:10.1021/i160032a009.

(c) 2016 by the authors; licensee Preprints, Basel, Switzerland. This article is an open access article distributed under the terms and conditions of the Creative Commons by Attribution (CC-BY) license (http://creativecommons.org/licenses/by/4.0/). 